\title{
Actitud hacia la Evaluación del Desempeño Docente y su Relación con la Autoevaluación del Propio Desempeño, en Profesores Básicos de Copiapó, La Serena y Coquimbo
}

\section{Attitude towards the Evaluation of Teaching Performance and their Relationship to One's Own Self-Performance in Teachers of Elementary Schools in Copiapó, La Serena, and Coquimbo}

\author{
Jorge Catalán y Mauricio González \\ Universidad de La Serena
}

\begin{abstract}
Se estudió la relación entre la actitud de los profesores hacia el proceso de evaluación de su docencia, impulsado por el Ministerio de Educación, y la forma en que ellos autoevalúan su propio desempeño docente. Se utilizó un diseño correlacional en el que participaron 509 profesores de segundo ciclo básico de las comunas de La Serena, Coquimbo y Copiapó. Se encontró que los profesores con una actitud positiva se autoevalúan más satisfactoriamente que los profesores con una actitud negativa, pero esta relación no es lineal. La edad se correlacionó directamente con la autoevaluación e inversamente con la actitud. Se infiere que los profesores más jóvenes tendrían mejor disposición a ser evaluados y a mejorar su desempeño a partir de estas evaluaciones.
\end{abstract}

Palabras claves: desempeño docente, evaluación del desempeño docente, autoevaluación del desempeño docente, desarrollo profesional.

\begin{abstract}
We studied the relationship between teachers' attitudes towards the evaluation of teaching performance, required by the Ministry of Education, and how they self-evaluate their own performance. The study used a correlational design in which 509 third and fourth grade teachers from the communities of La Serena, Coquimbo, and Copiapó participated. It was found that teachers with a positive attitude rate themselves higher in self-evaluation than those teachers with more negative attitudes. However, this relationship is not linear. We also found that age correlated directly with the self- evaluation and inversely with attitudes. It is suggested that younger teachers are more willing to be evaluated and improve their performance based on these evaluations.
\end{abstract}

Keywords: teaching performance, evaluation of teaching performance, self-evaluation of teaching performance, professional development.

Introducción

Desde el año 2003 se ha venido implementando el proceso de evaluación del desempeño docente en los establecimientos municipalizados chilenos. La puesta en marcha del sistema se realizó con 3.740 docentes del primer ciclo básico de 63 comunas del país, con la participación de los municipios y el Centro de Perfeccionamiento, Experimentación e Investigaciones Pedagógicas (CPEIP) del Ministerio de Educación
(MINEDUC) y con la asesoría de una institución independiente especializada en evaluación. En el año 2004 se inició el proceso en varias regiones del país, en cumplimiento con lo establecido por las leyes $19.993 \mathrm{del} 12$ de Febrero de 2004 (MINEDUC, 2004a, Febrero 12) y 19.961 del 9 de Agosto de 2004 (MINEDUC, 2004b, Agosto 9), en las que se establecen incentivos para profesores y las condiciones en las que se llevará a cabo el proceso, complementado con lo dispuesto en el Decreto $\mathrm{N}^{\circ}$ 192, del 30 de Agosto de

Jorge Catalán Ahumada, Departamento de Psicología, Universidad de La Serena, Coquimbo, Chile.

Mauricio González Arias, Departamento de Psicología, Universidad de La Serena, Coquimbo, Chile.

La correspondencia relativa a este artículo debe ser dirigida a Jorge Catalán Ahumada, Departamento de Psicología, Facultad de Humanidades, Universidad de La Serena, Matta 147, Coquimbo, Chile. E-mail: jcatalan@userena.cl El estudio fue financiado por la Dirección de Investigación de la Universidad de La Serena (Proyecto DIULS 0220-2-27) 
2004 (MINEDUC, 2004c, Agosto 30). Luego, en el año 2004 se incluyó a profesores de segundo ciclo básico y en el año 2005 se incorporó lenguaje y matemáticas de educación media. En las regiones de Atacama y Coquimbo el proceso se inició a partir del año 2004 y en el 2006 se continuó con el segundo ciclo.

La implementación de la evaluación docente implica un importante cambio que afecta las prácticas, creencias y sentimientos de los profesores, por lo que ellos se convierten en los principales actores de la implementación y, por lo tanto, resulta imprescindible tomarlos en cuenta a la hora de evaluar la viabilidad de la propuesta (De Vicente, 2002; Marcelo, 1999; Murillo, 1999).

Es razonable pensar que los profesores puedan tener ciertas reticencias a ser evaluados, por las posibles consecuencias que les pudieran traer sus resultados. De hecho, el Colegio de Profesores propuso un estatuto propio, cuando se produjo el traspaso de los colegios a los municipios, en el que relaciona la "evaluación" con "desempeño profesional", enunciando luego las dificultades que podría conllevar la evaluación docente (Colegio de Profesores A.G., 2000; Núñez, 2000). Si bien los profesores han estado representados en la comisión tripartita (Colegio de Profesores de Chile, Asociación Chilena de Municipalidades y Ministerio de Educación) que ha establecido las condiciones del proceso y disponen de información accesible sobre lo que se enfrentarán en su ejecución, ello no significa que sus creencias sobre la materia sean plenamente concordantes con los propósitos de la evaluación. Resulta, por tanto, relevante investigar lo que con ellos acontece, para que los directivos de los colegios y los organismos gubernamentales tengan mayor información para implementar el proceso y, muy especialmente, para aportar a los propios profesores elementos para su reflexión profesional y concebir la evaluación docente como una genuina instancia de mejoramiento personal, profesional e institucional.

El problema abordado en esta investigación se puede sintetizar en la siguiente pregunta: ¿Existe alguna relación entre la actitud de los profesores ante la evaluación del desempeño docente realizada por el MINEDUC y la autoevaluación que hacen de su propio desempeño? Para abordar esta interrogante, se planteó como objetivo general: Establecer la relación entre la actitud hacia la evaluación docente realizada por el MINEDUC y la autoevaluación del propio desempeño, en profesores del segundo ciclo básico de las comunas de Copiapó (región de Atacama), La Serena y Coquimbo (región de Coquimbo). Los objetivos específicos de este estudio fueron: a) Describir la actitud hacia la evaluación docente de los profesores y su relación con las variables sociodemográficas; b) Describir la autoevaluación que los docentes realizan de su desempeño y su relación con las variables sociodemográficas; y c) Describir la relación entre la actitud hacia la evaluación docente y la autoevaluación de su propio desempeño.

Además de profesores de colegios municipalizados, la inclusión en el estudio de profesores de establecimientos particulares subvencionados obedece a la conveniencia de tener otro referente para analizar los resultados, en virtud de dos situaciones que pudieran ser relevantes: por una parte, la postura crítica del Colegio de Profesores, entidad a la que pertenecen profesores de establecimientos de diferente tipo de dependencia administrativa y, por otra, la posible relación entre tener o no tener que someterse a evaluación y la actitud ante el proceso, como también la propia autoevaluación que los docentes realizan en una $u$ otra condición. La inclusión de tres comunas, una de ellas de una región diferente, también permite explorar diferencias en este ámbito.

\section{La Evaluación del Desempeño Docente como Instancia de Desarrollo Profesional}

El desarrollo profesional se ha conceptualizado de diversas formas, asociándolo a varios otros conceptos: perfeccionamiento de profesores, reciclaje de profesores, formación permanente, formación en servicio, desarrollo del profesorado. La más elemental y clara distinción parece ser la que diferencia entre el perfeccionamiento y el desarrollo profesional, en cuanto a que el perfeccionamiento está asociado a la intervención externa y el desarrollo profesional 
está caracterizado por una intervención interna o de la propia persona (De Vicente, 1992; Marcelo, 1999).

En lo sustantivo, el desarrollo profesional parece utilizarse en dos sentidos básicos: uno se relaciona con la adquisición de conocimientos, destrezas y estrategias para procesar información y para desarrollar la enseñanza; el otro, con el cambio continuo que el desempeño profesional experimenta con el tiempo, lo que permite que algunos autores propongan una progresión de etapas en este proceso (De Vicente, 2002).

Las orientaciones o pragmáticas que han guiado el desarrollo profesional de los docentes (Villar, 1991), permiten diferenciar tres modelos: dos que tienen como foco lo individual y uno con focalización en lo organizativo. Entre los individuales, uno se centra en la identificación y resolución de necesidades; otro, en la evaluación de la acción docente; el tercero está centrado en la metáfora del profesor investigador, que concibe al profesor como un profesional reflexivo.

En una revisión de los modelos, De Vicente (2002) identifica tres aspectos básicos en la formación de los profesores: conocimiento, reflexión y colaboración. Subyace a estos aspectos la concepción del profesor como un profesional reflexivo, colaborador y que construye conocimiento colectivamente (Hargreaves, 1996).

La posibilidad de intervención de los profesores en aspectos que contribuyan al mejoramiento de la calidad de la educación tiene como requerimiento que los propios profesores cambien y que este cambio surja de la evaluación de su quehacer y de lo que acontece en las instituciones en las que lo desarrollan (Cortés, 2007, Octubre; Murillo, 1999; Villar, 1995), de manera que:

...el desarrollo profesional del profesor necesita apoyarse en los resultados de la evaluación de su acción educativa, tanto a nivel específico como genérico; así, deberá conocerse su capacidad para dominar y aplicar las habilidades técnicas para la práctica; habrá de saberse hasta qué punto es capaz de incrementar la claridad, el significado y la coherencia a través de la práctica reflexiva; será necesario averiguar si está dotado del conocimiento suficiente y de la habilidad necesaria para la investigación y la exploración; si posee, en fin, la adecuada disposición a recibir y dar ideas, a prestar asistencia y a recibirla de otros, a la colaboración. (De Vicente, 1996, p. 299)

Las iniciativas para la evaluación del desempeño docente entroncan con el camino recorrido internacionalmente para el mejoramiento de la calidad de la educación. Es especialmente en la última década cuando se elaboran estándares de desempeño, cuya prioridad es el fortalecimiento de la profesión docente y el desarrollo profesional, como lo demuestra la labor realizada en este ámbito por diversos países, tales como Inglaterra, Estados Unidos, Canadá y Francia. Se trata de hacer mensurable u observable lo que los docentes deben saber y saben hacer en el ejercicio de su profesión, de modo que la generación de estos estándares obedece al propósito de contribuir a la calidad docente y, por ende, de la educación, como también a favorecer un reconocimiento social acorde con el status profesional del profesor.

En el caso chileno, se ha logrado llevar adelante una labor conjunta del MINEDUC, el Colegio de Profesores y la Asociación Chilena de Municipalidades, lo cual ha permitido fundar un sistema nacional de desempeño docente (Colegio de Profesores de Chile A.G., 2003), implementado en forma gradual, dada su complejidad y el requerimiento de numerosos evaluadores. La evaluación se realiza a los docentes del sistema municipal de educación (en Chile existe también la educación particular subvencionada, la particular pagada y la de corporaciones privadas o de administración delegada), siguiendo los criterios establecidos en el Marco para la Buena Enseñanza (MBE, MINEDUC, 2003), documento que busca contribuir a fortalecer la profesión docente y mejorar la calidad de la educación. El MBE contempla cuatro dominios que, en su conjunto, pretenden abordar los aspectos más relevantes del proceso educativo. El Domino A corresponde a la Preparación para la Enseñanza, el Dominio B, a la Creación de un Ambiente Propicio para el Aprendizaje, el Dominio C, a la Enseñanza 
para el Aprendizaje de todos los Estudiantes y el Domino D, a las Responsabilidades Profesionales. A cada una de estas dimensiones corresponden cinco o seis criterios de calidad verificables, con sus respectivos indicadores (MINEDUC, 2000; 2003).

Según Nevo (1997), existe una variedad de procedimientos e instrumentos para evaluar el desempeño docente. Entre estos últimos menciona tests de competencias, evaluaciones de experiencias de campo, escalas de puntuaciones, observaciones de clases, evaluación por los alumnos, evaluaciones por pares, resultados de los alumnos y autoevaluaciones.

En Chile, el MINEDUC (Colegio de Profesores de Chile A.G., 2003) ha propuesto los siguientes instrumentos:

1. Evidencias estructuradas, realizadas y reportadas por el propio profesor (portafolio).

2. Autoevaluación realizada de acuerdo a una pauta con las dimensiones de los criterios establecidos en el MBE. El instrumento recoge información cuantitativa y permite incorporar comentarios cualitativos.

3. Entrevista estructurada, basada en los dominios y criterios del MBE. Incluye preguntas sobre el contexto en que el/la profesor/a labora y que pudiera afectar su desempeño.

4. Informe de referencia de terceros, que permite complementar la información anterior, realizado por el Director o Jefe de Unidad Técnico Pedagógica del establecimiento del evaluado.

La información que se obtiene a partir de la evaluación se traduce en un informe que se le entrega en forma personal al docente con los aspectos más logrados y menos logrados, ubicándolo en alguno de los siguientes niveles: destacado, competente, básico o insatisfactorio. Los docentes correspondientes a las categorías destacado y competente tienen la posibilidad de recibir una asignación variable, para lo cual deben rendir una prueba de conocimientos disciplinarios y pedagógicos de su especialidad. Los docentes correspondientes a las categorías básico e insatisfactorio pueden acceder a planes gratuitos para superar sus debili- dades. A quienes obtengan por segunda vez un resultado insatisfactorio se le asigna un par tutor por un año; si el docente resulta insatisfactorio por tercera vez, debería abandonar el sistema (CPEIP, 2005).

Mediante la evaluación del desempeño docente, se intenta evaluar fundamentalmente la responsabilidad y el desarrollo profesional (Reyes, 2000). Consecuentemente, no es un fin en sí, sino un medio para mejorar la calidad de la educación (Arancibia, 2001; Reyes 2000). Por lo mismo, requiere de condiciones especiales que permitan aprovechar su potencial:

La evaluación del profesor, cuando se
produce en un contexto de confianza y
apoyo mutuo, inicia un ciclo de reflexión
y de autoevaluación tanto individual
como institucional. No solo proporciona
retroalimentación al individuo y a la
organización, sino que sirve para esti-
mular la reflexión acerca de las normas
y valores que apoyan la práctica profe-
sional. (De Vicente, 2002 , p. 125)

Alvarado (2000) sugiere un conjunto de características que debería poseer un proceso de evaluación del desempeño docente, entre estas: su integración a la evaluación de todo el sistema educativo, objetividad y asesoramiento a docentes, utilidad como sistema de promociones e incentivos, preparación y persuasión de los docentes para aceptar ser evaluados, como instancia para mejorar su desempeño (aspecto que viene a sustentar la relevancia de la presente investigación), consideración de las circunstancias y condiciones en que se ejerce la labor y el conocimiento confidencial de los resultados por parte de quien fue evaluado.

La evaluación del desempeño docente puede enfrentarse a diversas dificultades, especialmente relacionadas con aspectos que puedan representar amenazas para los profesores, estabilidad laboral, problemas de recursos, objetividad, tensiones y fricciones entre profesores y directivos, facilitación del ajuste a lo dispuesto por directivos (Colegio de Profesores de Chile A.G., 2000). En particular, la autoevaluación del desempeño académico (incorporada dentro del proceso de evaluación en uso y utilizada en el estudio que se reporta), si bien estimula la 
reflexión (Elliot, 1996), presenta también algunas dificultades; entre otras, constituirse en una forma de auto-justificación para quienes tengan bajos desempeños, que podría hacer que una persona incompetente no advierta su mal rendimiento y, por otra parte, la menor exactitud para evaluarse de los menos competentes (Nieto, 1996). Yániz (1998) plantea que las limitaciones de la autoevaluación se relacionan con dos tipos de creencias: los deseos de autorrealizarse, que provocan un importante error en los resultados, y la incapacidad de los profesores de analizar su quehacer en forma objetiva, lo que se traduce en imprecisión a la hora de autoevaluarse.

Las Actitudes de los Profesores y la Evaluación del Desempeño Docente

Una manera de aproximarse a la forma como los profesores podrían enfrentar el proceso de evaluación de su desempeño es mediante el conocimiento de sus actitudes, entendidas como "disposiciones permanentes de ánimo formadas por un conjunto de convicciones y sentimientos que llevan al sujeto que las tiene a actuar y expresarse según ellas en sus actos y opiniones" (Sierra Bravo, 1999, p. 371). Al revisar una serie de definiciones, Chávez, Tron y Vaquero (1992) concluyen que "una actitud ha sido considerada como un estado disposicional, como una respuesta emotiva o como una tendencia a comportarse positiva o negativamente" (p. 777).

De esta manera, se puede señalar que las actitudes comprenden tres factores: a) un factor ideológico, formado por ideas y convicciones determinadas, que también ha sido concebido como un componente cognitivo, e incluye las creencias, expectativas e información general que las personas manejan sobre el objeto o situación; b) un factor sentimental, que implica simpatía o antipatía hacia cosas o personas, concebido como un componente afectivo, ya que se asocia con los sentimientos positivos o negativos que experimenta una persona frente a un objeto o situación; y c) un factor reactivo, que impulsa a obrar en las distintas situaciones sociales en consonancia con las convicciones y sentimientos profesados, el que, dependiendo del énfasis conceptual, también se ha denominado componente comportamental o de tendencia a la acción. La importancia que cada uno de estos factores ha tenido en la definición de la actitud ha variado a lo largo del tiempo y de los autores. Inicialmente, se puso mucho énfasis en el aspecto conductual, pues se esperaba que la actitud fuera un buen predictor de la conducta, pero cuando la investigación demostró una débil correlación entre ambas, el énfasis cambió al aspecto afectivo. Con el desarrollo del paradigma cognitivo se ha comenzado a valorar nuevamente el aspecto cognitivo y a buscar procedimientos alternativos para su medición (Morera \& Rodríguez, 1994).

Chávez et al. (1992) proponen dos estrategias fundamentales para medir actitudes: la observación y la utilización de escalas de medición. La mayor ventaja de las escalas es que proporcionan mayor precisión y mayor posibilidad de aplicación de las matemáticas a su análisis y estudio (Sierra Bravo, 1999). Esto se realiza con escalas de diferencial semántico (Arce, Stem, Andrade \& Seoane, 1997), escalas de Likert y las denominadas actitudes implícitas (Briñol, Horcajo, Becerra, Falces \& Sierra, 2002).

Algunos estudios comparativos han demostrado que las escalas Likert permiten obtener, con un alto grado de validez y confiabilidad, los mismos resultados de las otras escalas, pero cuya confección resulta más laboriosa (Chávez et al., 1992) o requieren muestras más grandes y mayores conocimientos técnicos para su elaboración (Asún \& Zúñiga, 2008). En la actualidad las escalas de actitudes tienen una gran aplicación en las investigaciones sociales, bien de manera independiente, bien formando parte de cuestionarios simples o de entrevistas (Sierra Bravo, 1999).

Por lo anterior, se puede señalar que es pertinente y viable estudiar las actitudes de los profesores hacia su propia evaluación del desempeño. De esta manera, en términos generales se hipotetiza que los profesores que tienden a autoevaluar mejor su desempeño, tienden también a presentar una mejor actitud ante la evaluación del desempeño. Si bien el diseño de este estudio no permite sacar conclusiones causales, los resultados permitirán discutir la 
posibilidad que una mala o buena actitud pueda llevar a los profesores a aceptar o a rechazar la evaluación docente, o bien que la percepción a priori que los profesores tienen de su propio desempeño pueda generar temor o desconfianza ante la evaluación y, por consiguiente, una mala actitud.

\section{Método}

La investigación se desarrolló de acuerdo a un diseño transversal correlacional. En una fase preliminar se describieron las variables en estudio, para posteriormente determinar la posible existencia de asociación entre ellas.

\section{Muestra}

Se seleccionó una muestra probabilística bietápica de la población de profesores/as de segundo ciclo de educación básica de los colegios municipalizados y particulares subvencionados de las comunas de Copiapó, La Serena y Coquimbo. A partir de una tabla que determina el tamaño de una muestra extraída de una población finita (Arkin \& Colton, citado en Sierra Bravo, 1999), se estimó un tamaño provisorio de la muestra, considerando un margen de error de $5 \%$ para las actitudes hacia la evaluación del desempeño docente y para la autoevaluación del desempeño docente, con un nivel de confianza de $95 \%$. Sin embargo, como es posible que se produzca un efecto de diseño al seleccionar la muestra por etapas y al no contar con información para su cálculo sobre la variabilidad de las variables señaladas, se optó por aumentar el tamaño muestral provisorio en un $40 \%$.

En la primera etapa se estratificó la población por comuna y tipo de dependencia (municipalizada y particular subvencionada), seleccionando al azar 23 colegios de los seis estratos resultantes, procurando mantener la proporcionalidad por comuna y tipo de dependencia de la población. La segunda etapa consistió en la selección al azar de profesores de cada uno de los colegios seleccionados, con un proceso de afijación simple.

Se seleccionaron al azar colegios y profesores adicionales en cada etapa como reemplazo en caso necesario. La muestra quedó constituida por 509 profesores, distribuidos como se muestra en la Tabla 1.

\section{Instrumentos}

Escala de actitudes hacia la evaluación del desempeño docente (AHED). Para cumplir con el primer objetivo específico de este estudio, en una tesis de pregrado se elaboró una escala Likert que permitiera medir las actitudes de los profesores hacia la evaluación del desempeño (Cerda, Cortés, Mejías \& Milla, 2006). El instrumento definitivo cuenta con 30 ítems positivos y negativos, con cinco categorías de respuesta (muy de acuerdo, de acuerdo, ni de acuerdo ni en desacuerdo, en desacuerdo y muy en desacuerdo). El instrumento fue diseñado considerando las dimensiones clásicas de las actitudes (cognitiva, afectiva y conductual) y de acuerdo a las dimensiones de la evaluación docente. El puntaje teórico máximo de las escala es de 150 puntos, el mínimo de 30 y la media de 90. Los puntajes altos se interpretan como una actitud positiva o favorable y los puntajes bajos como una actitud negativa o desfavorable. Se recurrió al juicio de expertos para asegurar la validez de contenido de la escala. La validación del instrumento se realizó con una muestra piloto de 100 profesores/as de La Serena y Coquimbo. En cuanto a la validez de constructo, un análisis de componentes principales reveló que un $51,4 \%$ de la varianza total de las actitudes hacia la evaluación del desempeño docente se concentra en un solo factor, lo que indicaría que la escala estaría midiendo una actitud general. La obtención de un coeficiente alfa de Cronbach de 0,936 para el total de la escala da cuenta que el instrumento posee consistencia interna. Los valores de alfa obtenidos para cada una de las dimensiones fueron: conductual, 0,846; cognitiva, 0,875; afectiva, 0,787.

Escala de autoevaluación del desempeño docente. Es una escala tipo Likert que cuenta con 40 afirmaciones referidas a las cuatro dimensiones del MBE: a) preparación para la enseñanza, referida a la organización del contenido en función del aprendizaje del estudiante; b) creación de un ambiente propicio para el aprendizaje de los 
Tabla 1

Caracterización de la Muestra

\begin{tabular}{|c|c|c|}
\hline Variable & $N$ & $\%$ \\
\hline \multicolumn{3}{|l|}{ Sexo } \\
\hline Mujer & 352 & 69,2 \\
\hline Hombre & 157 & 30,8 \\
\hline Total válidos & 509 & 100,0 \\
\hline \multicolumn{3}{|l|}{ Edad } \\
\hline 20 a 35 años & 142 & 28,3 \\
\hline 36 a 50 años & 201 & 40,1 \\
\hline 51 a 70 años & 158 & 31,5 \\
\hline Total válidos & 501 & 99,9 \\
\hline \multicolumn{3}{|l|}{ Tipo de Dependencia } \\
\hline Municipal & 278 & 54,6 \\
\hline Particular Subvencionado & 231 & 45,4 \\
\hline Total válidos & 509 & 100,0 \\
\hline \multicolumn{3}{|l|}{ Comuna } \\
\hline La Serena & 169 & 33,2 \\
\hline Coquimbo & 140 & 27,5 \\
\hline Copiapó & 200 & 39,3 \\
\hline Total válidos & 509 & 100,0 \\
\hline \multicolumn{3}{|l|}{ Región } \\
\hline Coquimbo & 309 & 60,7 \\
\hline Atacama & 200 & 39,3 \\
\hline Total válidos & 509 & 100,0 \\
\hline \multicolumn{3}{|l|}{ Años de Servicio } \\
\hline 1 a 10 años & 329 & 64,8 \\
\hline 11 a 20 años & 82 & 16,1 \\
\hline 21 a 30 años & 47 & 9,3 \\
\hline 31 a 45 años & 50 & 9,8 \\
\hline Total válidos & 508 & 100,0 \\
\hline
\end{tabular}

alumnos; c) enseñanza para el aprendizaje de los alumnos y d) profesionalismo docente (MINEDUC, 2003). Las afirmaciones tienen cuatro alternativas de respuesta (nunca, ocasionalmente, generalmente, siempre). El puntaje teórico máximo de la escala es de 160, el mínimo de 40 y la media de 100. Mientras más alto el puntaje, más satisfactoria es la autoevaluación. La validez de contenido se obtuvo por medio del juicio de expertos, quienes evaluaron el ajuste de los reactivos a las dimensiones señaladas. En la estimación de la consistencia interna del instrumento se obtuvo un coeficiente alfa de Cronbach de 0,88 para el total, oscilando entre 0,69 y 0,74 en sus dimensiones. Para estimar la estabilidad temporal del instru- mento se calculó el coeficiente $r$ de Pearson test-retest, el que fue de 0,85 para el instrumento total (Cepeda, Espinoza \& Rojas, 2003).

\section{Procedimiento}

Los instrumentos fueron aplicados de manera colectiva por los investigadores y ayudantes en los propios establecimientos seleccionados, con el consentimiento de los profesores y tomando los resguardos correspondientes para asegurar la confidencialidad de la información. Se informó a los profesores, de manera general, que se estaba realizando una investigación sobre lo que los profesores piensan del proceso de eva- 
luación y la manera como ellos se autoevalúan. La investigación fue conocida y aprobada por el Comité de Ética de la Facultad de Humanidades a la que se adscriben los investigadores.

En todos los colegios se aplicó primero la escala de actitud y a continuación la autoevaluación, de manera que la autoevaluación no sesgara la medición de la actitud.

Para conseguir la objetividad de la aplicación, se realizó una capacitación acerca de los instrumentos a todos los administradores.

\section{Resultados}

Actitud hacia la Evaluación del Desempeño Docente y su Relación con Variables Sociodemográficas

En la muestra total se encontró una actitud hacia la evaluación del desempeño levemente positiva $(M=94,66 ; D E=21,40)$ respecto del puntaje teórico medio $(M=90)$, siendo la distribución tendiente a la normal (véase la Figura 1).

De acuerdo al sexo, el análisis de varianza no mostró una diferencia significativa entre los promedios de hombres y mujeres $(F[1,507]=0,33, p=0,57)$. Respecto de la edad de los profesores, el análisis de varianza mostró diferencias significativas de los promedios de la escala $(F[2,498]=$ $5,11, p=0,006)$, encontrándose que la actitud del grupo de 20 a 35 años $(M=99,33$; $D E=18,64)$ es más positiva que la del grupo de 51 a 70 años $(M=91,66 ; D E=22,57)$. El grupo de 36 a 50 años $(M=94,47 ; D E=$ $21,14)$ no presentó diferencias significativas con las demás edades. Respecto del tipo de dependencia, el análisis de varianza mostró diferencias significativas de los promedios de la escala $(F[1,507]=55,75, p=0,000)$, encontrándose que los profesores de los colegios municipalizados presentan una actitud más negativa $(M=88,5 ; D E=22,69)$ que los profesores de los colegios particulares subvencionados $(M=102,05 ; D E=17,08)$.

Con relación a la comuna donde se ubica el colegio en el que trabaja el profesor, el análisis de varianza mostró una diferencia significativa de los promedios de la escala $(F[2,506]=3,11, p=0,046)$ y el análisis post hoc (Tukey) mostró que los profesores de La Serena tienen una actitud significativamente más positiva $(M=97,50 ; D E=$ $21,93)$ que los de Coquimbo $(M=91,44 ; D E$ $=22,55)$, pero no hubo diferencia significativa del promedio de estos respecto del de los profesores de Copiapó $(M=94,53 ; D E$ $=19,85$ ), cuyo promedio es similar al promedio total de la muestra. Por otro lado, no se encontraron diferencias significativas en

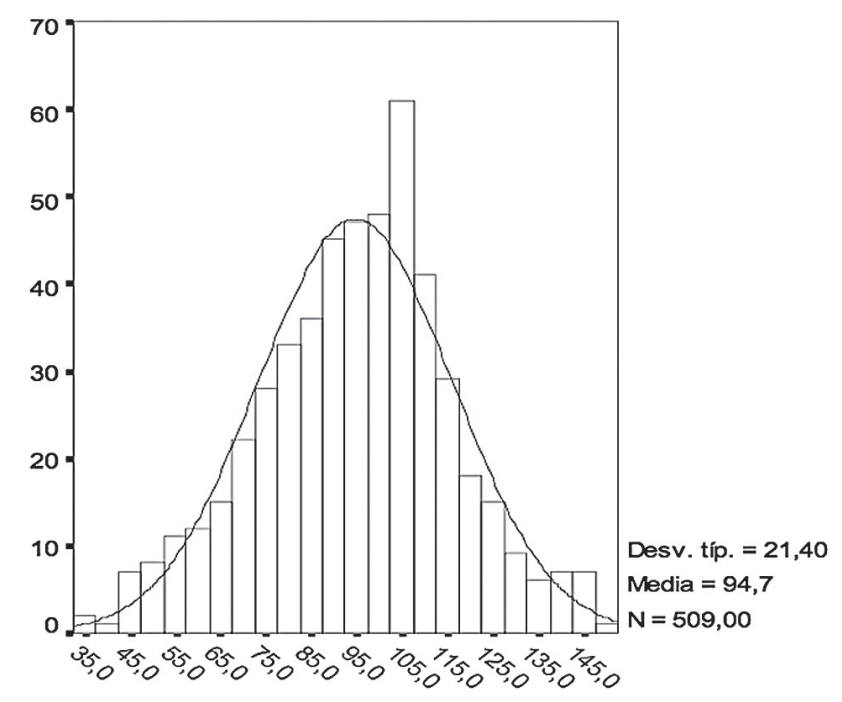

Figura 1. Histograma de los puntajes de actitud hacia la evaluación del desempeño docente. 
el promedio de actitudes según las regiones $(F[1,507]=0,01, p=0,906)$ ni según los años de servicio $(F[3,504]=2,23, p=0,083)$ (véase la Tabla 2). Al calcular el coeficiente de correlación lineal entre la actitud hacia la evaluación del desempeño y la edad, se encontró una débil correlación inversa, $r(301)=-0,201, p=0,000$.

Si bien no era uno de los objetivos del estudio, resulta relevante destacar que existe una asociación entre la edad y el tipo de dependencia, de modo que los profesores más jóvenes se encontraban mayoritaria y significativamente en los colegios particulares subvencionados y los profesores de mayor edad, en los colegios municipales, $\chi^{2}(2$,
$N=301^{1}$ ) $=62,6, p=0,000$ (véase la Tabla 3). Debido a esto, se recalculó el coeficiente de correlación controlando el tipo de dependencia. La correlación parcial obtenida para la actitud hacia la evaluación del desempeño y la edad mostró una correlación no significativa, $r(298)=-0,051, p=0,370$.

1 Este análisis se hizo solamente para los profesores de Coquimbo y La Serena, puesto que para Copiapó solo se consignó la edad en categorías, lo que impide tratarla como una variable intervalar. De esta manera, los reportes de correlaciones con la edad excluyen a los casos de Copiapó.

Tabla 2

Descriptivos de la Variable Actitud hacia la Evaluación del Desempeño Docente

\begin{tabular}{|c|c|c|c|c|c|c|c|}
\hline Variable & Categorías & $N$ & Media & $\begin{array}{c}\text { Desviación } \\
\text { estándar }\end{array}$ & $\begin{array}{l}\text { Error } \\
\text { típico }\end{array}$ & Mínimo & Máximo \\
\hline \multicolumn{8}{|l|}{ Sexo } \\
\hline & Hombre & 352 & 94,30 & 22,05 & 1,18 & 33 & 149 \\
\hline & Mujer & 157 & 95,48 & 19,94 & 1,59 & 44 & 146 \\
\hline \multicolumn{8}{|l|}{ Edad } \\
\hline & 20 a 35 & 142 & 99,33 & 18,64 & 1,56 & 44 & 144 \\
\hline & 36 a 50 & 201 & 94,47 & 21,14 & 1,49 & 36 & 149 \\
\hline & 51 a 70 & 158 & 91,66 & 22,57 & 1,80 & 33 & 146 \\
\hline \multicolumn{8}{|c|}{ Tipo de Dependencia } \\
\hline & Municipal & 278 & 88,53 & 22,69 & 1,36 & 33 & 146 \\
\hline & P. Subvencionado & 231 & 102,05 & 17,08 & 1,12 & 51 & 149 \\
\hline \multicolumn{8}{|l|}{ Comuna } \\
\hline & La Serena & 169 & 97,50 & 21,93 & 1,69 & 40 & 149 \\
\hline & Coquimbo & 140 & 91,44 & 22,55 & 1,91 & 33 & 146 \\
\hline & Copiapó & 200 & 94,53 & 19,85 & 1,40 & 46 & 144 \\
\hline \multicolumn{8}{|l|}{ Región } \\
\hline & Coquimbo & 309 & 94,75 & 22,38 & 1,27 & 33 & 149 \\
\hline & Atacama & 200 & 94,53 & 19,85 & 1,40 & 46 & 144 \\
\hline \multicolumn{8}{|c|}{ Años de Servicio } \\
\hline & 1 a 10 años & 329 & 96,25 & 19,74 & 1,09 & 44 & 144 \\
\hline & 11 a 20 años & 82 & 94,12 & 23,57 & 2,60 & 40 & 149 \\
\hline & 21 a 30 años & 47 & 89,91 & 24,90 & 3,63 & 36 & 143 \\
\hline & 31 a 45 años & 50 & 89,88 & 23,95 & 3,39 & 33 & 146 \\
\hline Total & & 509 & 94,66 & 21,40 & 0,95 & 33 & 149 \\
\hline
\end{tabular}


Tabla 3

Distribución Relativa de la Edad y Tipo de Dependencia de los Profesores

\begin{tabular}{lrcrr}
\hline \multirow{2}{*}{ Tipo de Dependencia } & \multicolumn{3}{c}{ Edad (en años) } & \multirow{2}{*}{ Total } \\
\cline { 2 - 4 } & 20 a 35 & 36 a 50 & 51 a 70 & \\
\hline Municipal & 6,6 & 15,9 & 22,3 & 44,9 \\
Particular Subvencionado & 23,3 & 26,2 & 5,6 & 55,1 \\
Total & 29,9 & 42,2 & 27,9 & 100,0 \\
\hline
\end{tabular}

Autoevaluación del Propio Desempeño

Docente y su Relación con Variables Sociodemográficas

El promedio de la autoevaluación de la muestra estudiada fue de 143,65 ( $D E$ $=10,69)$, el cual se ubica por encima del promedio teórico de 100 puntos y cerca del puntaje máximo teórico de 160 puntos. Adicionalmente, no se encuentran puntajes mínimos inferiores al promedio teórico. De esta manera, la distribución de los puntajes de autoevaluación tiene una fuerte asimetría negativa, es decir, todos los profesores tienden a autoevaluarse satisfactoriamente (véase la Figura 2).
De acuerdo al sexo, el análisis de varianza mostró una diferencia significativa de la autoevaluación $(F[1,507]=6,37, p$ $=0,012)$ entre los promedios de hombres $(M=144,45 ; D E=10,25)$ y de mujeres $(M$ $=141,87 ; D E=11,47)$. Con relación a la edad, se encontraron diferencias significativas en los promedios de autoevaluación a partir de un análisis de varianza $(F[2$, $498]=8,50, p=0,000)$ y el análisis post hoc (Tukey) mostró que el grupo de edades de 20 a 35 años $(M=142,16 ; D E=10,26)$ presenta una autoevaluación de su propio desempeño menos satisfactoria que el grupo de edades de 51 a 70 años $(M=146,42 ; D E=10,13)$, pero no mostró diferencias entre estos dos

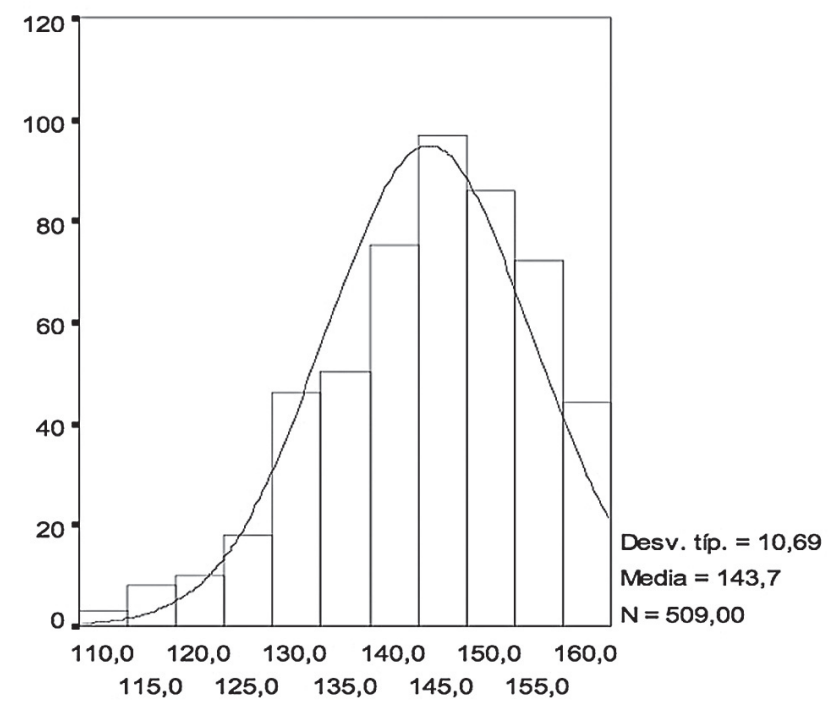

Figura 2. Histograma de los puntajes de autoevaluación. 
grupos y el grupo con edades de 36 a 50 años. De acuerdo al tipo de dependencia, el análisis de varianza mostró diferencias significativas entre los promedios $(F[1,507]$ $=22,75, p=0,000)$, encontrándose que los profesores de los colegios municipales ( $M$ $=145,67 ; D E=11,07)$ se autoevalúan más satisfactoriamente que los profesores de los colegios particulares subvencionados $(M=$ $141,23 ; D E=9,71)$. Respecto de la comuna, no se encontraron diferencias significativas en el promedio de autoevaluación de los profesores de La Serena, Coquimbo y Copiapó $(F[2,506]=2,31, p=0,100)$. De la misma manera, no se encontraron diferencias significativas en la autoevaluación respecto de las regiones estudiadas $(F[1,507]$ $=2,60, p=0,107$ ) (ver la Tabla 4). Por otro lado, de acuerdo a los años de servicio, el análisis de varianza mostró diferencias significativas $(F[3,504]=7,23, p=0,000)$ y el análisis post hoc (Tukey) mostró que el grupo de 1 a 10 años de servicio $(M=142,64$; $D E=10,87)$ presenta una autoevaluación más baja respecto a los grupos de 21 a 30 años $(M=147,37 ; D E=7,83)$ y de 31 a 45 años $(M=143,65 ; D E=10,69)$, pero no presenta diferencias con el grupo de 11 a 20 años $(M=142,35 ; D E=11,33)$. Este último grupo de 11 a 20 años, a su vez, presenta un promedio de autoevaluación significativamente menor que los grupo de 21 a 30 y de 31 a 45 años de servicio. Al calcular la correlación lineal entre la autoevaluación y la edad, se encontró una baja correlación directa, $r(301)=204, p=0,000$. Al calcular una correlación parcial entre la autoevaluación del desempeño y la edad, controlando

Tabla 4

Descriptivos de la Variable Autoevaluación del Desempeño Docente

\begin{tabular}{|c|c|c|c|c|c|c|c|}
\hline Variable & Categorías & $N$ & Media & $\begin{array}{l}\text { Desviación } \\
\text { estándar }\end{array}$ & $\begin{array}{l}\text { Error } \\
\text { típico }\end{array}$ & Mínimo & Máximo \\
\hline \multicolumn{8}{|l|}{ Sexo } \\
\hline & Hombre & 352 & 144,45 & 10,25 & 0,55 & 112 & 160 \\
\hline & Mujer & 157 & 141,87 & 11,47 & 0,92 & 111 & 160 \\
\hline \multicolumn{8}{|l|}{ Edad } \\
\hline & 20 a 35 & 142 & 142,16 & 10,26 & 0,86 & 112 & 160 \\
\hline & 36 a 50 & 201 & 142,30 & 11,07 & 0,78 & 111 & 160 \\
\hline & 51 a 70 & 158 & 146,42 & 10,13 & 0,81 & 114 & 160 \\
\hline \multicolumn{8}{|c|}{ Tipo de Dependencia } \\
\hline & Municipal & 278 & 145,67 & 11,07 & 0,66 & 112 & 160 \\
\hline & P. Subvencionado & 231 & 141,23 & 9,71 & 0,64 & 111 & 160 \\
\hline \multicolumn{8}{|l|}{ Comuna } \\
\hline & La Serena & 169 & 145,05 & 10,81 & 0,83 & 112 & 160 \\
\hline & Coquimbo & 140 & 143,32 & 9,42 & 0,80 & 111 & 160 \\
\hline & Copiapó & 200 & 142,71 & 11,34 & 0,80 & 113 & 160 \\
\hline \multicolumn{8}{|l|}{ Región } \\
\hline & Coquimbo & 309 & 144,27 & 10,23 & 0,58 & 111 & 160 \\
\hline & Atacama & 200 & 142,71 & 11,34 & 0,80 & 113 & 160 \\
\hline \multicolumn{8}{|c|}{ Años de Servicio } \\
\hline & 1 a 10 años & 329 & 142,64 & 10,87 & 0,60 & 111 & 160 \\
\hline & 11 a 20 años & 82 & 142,35 & 11,33 & 1,25 & 116 & 160 \\
\hline & 21 a 30 años & 47 & 147,37 & 8,74 & 1,28 & 127 & 160 \\
\hline & 31 a 45 años & 50 & 148,40 & 7,83 & 1,11 & 132 & 160 \\
\hline Total & & 509 & 143,65 & 10,69 & 0,47 & 111 & 160 \\
\hline
\end{tabular}


por el tipo de dependencia, no se encuentra una relación significativa, $r(298)=0,113, p$ $=0,051$.

\section{Relación entre la Actitud hacia la Evaluación del Desempeño Docente y la Autoevaluación del Propio Desempeño}

El coeficiente de correlación lineal de Pearson entre la actitud hacia la evaluación del desempeño docente y la autoevaluación del propio desempeño no fue significativo, $r(509)=0,037, p=0,399$. Solo se encontró una débil correlación directa entre la dimensión de profesionalismo docente de la autoevaluación y el componente cognitivo de la actitud, $r(509)=0,088, p=0,048$. No obstante lo anterior, se encontró una diferencia significativa al comparar los promedios de autoevaluación entre grupos extremos de profesores que tenían una actitud más positiva y los que tenían una actitud más negativa, a partir del primero y último quintil de la muestra. Los profesores del grupo de actitud negativa presentaron un puntaje promedio de autoevaluación de $144(D E=11)$, significativamente más bajo que los profesores con actitud positiva, cuyo promedio de autoevaluación fue de 147 ( $D E$ $=9), t(200)=-1,82, p=0,03$ (unilateral).

Debido a que se encontró una asociación entre la edad y el tipo de dependencia y que la edad está correlacionada con la actitud y la autoevaluación, se calcularon nuevos coeficientes de correlación entre la actitud hacia la evaluación del desempeño y la autoevaluación del propio desempeño, según el tipo de dependencia. En los profesores de colegios municipales, no se encontró relación entre ambas variables, $r(140)=0,088$, $p=0,303$. En cambio, en los profesores de colegios particulares subvencionados se encontró una correlación directa y débil, $r$ (169) $=0,309, p=0,000$. A su vez, el coeficiente de correlación parcial entre las variables actitud y autoevaluación, controlado por el tipo de establecimiento, fue significativo y directo, aunque débil, $r(298)=0,194, p=0,001$.

\section{Discusión}

Los resultados obtenidos muestran que los profesores con una actitud muy positi- va hacia la evaluación de desempeño se autoevalúan mejor que los profesores con una actitud muy negativa, aunque esta relación no se encuentra cuando se calcula un coeficiente de correlación entre ambas variables. Probablemente, esto se debió a la poca capacidad para discriminar que mostró el instrumento de autoevaluación, ya que todos los profesores se autoevaluaron satisfactoriamente. Estos resultados no son categóricos para mostrar una relación lineal entre la actitud hacia la evaluación del desempeño y la autoevaluación del propio desempeño en los profesores.

La dificultad de la escala de autoevaluación del propio desempeño para discriminar claramente entre los profesores no se explicaría solamente por dificultades técnicas en la confiabilidad de la escala, sino que por problemas de validez, en cuanto a la real posibilidad de acceder por medio de un autorreporte a un juicio más objetivo del propio desempeño. En este sentido, es interesante preguntarse si la tendencia de los profesores a autoevaluarse en extremo satisfactoriamente se debe a que en realidad lo hacen bien o a una baja capacidad de autocrítica y reflexión respecto de su ejercicio profesional y/o en una respuesta defensiva, no necesariamente consciente, ante una situación de evaluación que les genera temor y desconfianza. De esta manera, es válido suponer que un profesional más reflexivo y autocrítico tenga una autoevaluación menos satisfactoria, pero un mejor rendimiento a partir de una hetero-evaluación más objetiva, ya que alguien que considera que lo hace todo bien no tendría una predisposición a un perfeccionamiento continuo; en cambio, sí la tendría alguien que permanentemente realiza una reflexión crítica. Por otro lado, no resulta extraño que la única correlación lineal directa encontrada, aunque débil, fue entre la subescala de profesionalismo docente de la autoevaluación y la dimensión cognitiva de la actitud, puesto que ambos tópicos se relacionan más estrechamente con aspectos conscientes relacionados con el deber ser.

Independientemente de estos resultados, se sugiere desarrollar alguna intervención para mejorar la disposición de los profesores a involucrarse en el sistema de 
evaluación del desempeño docente. Esto último, a su vez, requiere de la generación de condiciones de confianza y apoyo, lo que no significa que el docente pierda protagonismo sino que, por el contrario, se sienta reconocido como profesional y se le conceda el tiempo y los espacios para reflexionar en torno a su quehacer (De Vicente, 2002).

Adicionalmente, se evidenció la existencia de una correlación de la edad con la actitud hacia la evaluación del desempeño y con la autoevaluación del propio desempeño. Los profesores más jóvenes tienen una actitud más positiva hacia la evaluación y una autoevaluación menos satisfactoria. Es válido suponer que los profesores jóvenes tendrían menos experiencia y estarían más abiertos a nuevos aprendizajes en el marco de su desempeño profesional. Esta apertura les permitiría una actitud diferente frente a su propia autoevaluación y sobre todo a una hetero-evaluación, ya que la verían como una retroalimentación necesaria para un proceso de aprendizaje y mejoramiento continuo de su docencia. Sería interesante corroborar estos resultados a partir de los datos recopilados hasta la fecha por el sistema de evaluación docente.

Por otro lado, llama la atención que los profesores de colegios municipales tengan puntuaciones en la actitud hacia la evaluación del desempeño docente significativamente más bajas y puntuaciones en autoevaluación significativamente más altas que los profesores de colegios particulares subvencionados, especialmente porque los primeros son los que se someten, por ley, a la evaluación y los segundos no. Una explicación posible está en el hecho que los profesores jóvenes se encuentran predominantemente en los colegios particulares subvencionados y son justamente los que tienen una actitud más positiva y una autoevaluación menos satisfactoria que los profesores de más edad. Otra potencial explicación es que los profesores municipalizados se autoevalúan mejor y están menos dispuestos a ser evaluados porque tienen aprensiones o se sienten amenazados por el proceso de evaluación.

Por lo anterior, fue necesario examinar nuevamente la relación entre la actitud hacia la evaluación del desempeño y la autoevaluación del propio desempeño, pero controlando el efecto de la edad y del tipo de dependencia. De esta manera, la correlación parcial entre la actitud hacia la evaluación del desempeño y la autoevaluación del propio desempeño resultó, aunque débil, directa y significativa, y al calcular las correlaciones separadamente por dependencia, solo se encontró correlación en los colegios de dependencia particular subvencionada, justamente donde se encuentran los profesores más jóvenes. Queda abierta la pregunta si esto se debe al efecto de la dependencia, en el sentido que los colegios particulares subvencionados no están sometidos a evaluación y, por lo mismo, sus profesores no se sentirían amenazados al responder a la autoevaluación, o bien, a mayor edad los profesores tienden a ser más autocomplacientes con sus evaluaciones.

En los resultados del proceso de evaluación docente del año 2008 (MINEDUC, 2009, Marzo 24) se muestra que los profesores con mejor desempeño se encuentran entre los 35 y los 49 años. Por el contrario, los profesores de menor y mayor edad tienen un desempeño más bajo, pero no se puede saber si a pesar de compartir esta similitud existe alguna diferencia importante entre ellos. Usando las mediciones ya realizadas en el programa de evaluación docente, sería interesante investigar la relación entre edad de los profesores y los resultados de sus autoevaluaciones y de las diferentes heteroevaluaciones, para contrastar la hipótesis que profesores más jóvenes con autoevaluaciones más bajas tienden a obtener mayores puntuaciones en las hetero-evaluaciones.

Si se logra el desarrollo de una cultura evaluativa en los profesores, los resultados de sucesivos procesos pueden ser un incentivo adicional, ya que dan cuenta que los profesores de desempeño insatisfactorio o básico, que se han enfrentado a una nueva evaluación, mejoran significativamente sus evaluaciones anteriores, en tanto que los de rendimientos más altos tienden a mantenerlos (CPEIP, 2008).

En el proceso que se está llevando a cabo en Chile se han considerado aspectos técnicos y gremiales; pero aún falta incorporar aspectos propios de la subjetividad de los actores, como también otorgarle mayor relevancia a la puesta en común de aspec- 
tos más propiamente profesionales relacionados con la evaluación, como es el caso del aprendizaje profesional esperable, aspecto crucial como sustento de la evaluación docente (Danielson, 2007, Octubre).

El hecho que el sistema de evaluación contemple la existencia de pares evaluadores, cuya cifra se incrementa de año en año, puede contribuir a desmitificar el proceso, tanto por la experiencia que personalmente cada evaluador par obtiene, como por el impacto que pueden tener al comunicarla en su interacción con otros profesores. Por otra parte, un aspecto muy importante es la posibilidad que tienen los evaluadores de conocer directamente la rigurosidad, objetividad y confidencialidad en la corrección de los instrumentos de evaluación.

Existen algunos recursos que claramente pueden aportar a involucrar más a los docentes. Uno de ellos es la posibilidad que los profesores emitan opiniones acerca del proceso de evaluación, lo que se lleva a cabo mediante un cuestionario complementario elaborado específicamente para tal propósito, cuyos resultados son accesibles, y actualmente dan cuenta de la valoración positiva que los docentes hacen del uso del portafolio (Manzi, 2007, Octubre). Del mismo modo, el MINEDUC proporciona información acuciosa y actualizada acerca del sistema de evaluación y de los resultados obtenidos cada año, pudiendo aprovecharse mejor este recurso, si se promueven acciones para que en cada establecimiento los profesores revisen ese material permanentemente, se lleven a cabo reflexiones al respecto y se informe y comente acerca de los resultados. Algo similar debería ocurrir en las instituciones formadoras de profesores. Un cambio cultural no se produce abruptamente. Las futuras generaciones de profesores deberían apropiarse tempranamente de la cultura evaluativa.

Los hallazgos de este estudio vienen a confirmar la necesidad de incrementar los esfuerzos en aspectos subjetivos e intersubjetivos de los sujetos de la evaluación. El proceso en sí paulatinamente ha empezado a exhibir sus bondades, como, por ejemplo, poner en evidencia que, dado un conjunto de variables que anticipan resultados negativos para grupos desfavorecidos, la labor pedagógica de un profesor de alto desempeño puede hacer la diferencia y obtener mejores rendimientos de sus estudiantes. No se trata, pues, que el profesor deficiente haga a un alumno de condiciones precarias deficiente sino que, ante un destino escolar casi inevitablemente deficiente, el profesor de calidad pueda revertir el presagio.

Sin duda, queda bastante camino por recorrer para que la evaluación docente aporte sustantivamente a la calidad de la educación chilena y al desarrollo profesional de sus profesores y profesoras. Bien vale, entonces, intentar avanzar en el sentido correcto.

\section{Referencias}

Alvarado, J. (2000). Construcción y evaluación de instrumentos de evaluación docente. Tesis no publicada para optar al grado de Magíster en Educación, Mención Administración Educacional, Pontificia Universidad Católica de Chile, Santiago, Chile.

Arancibia, V. (2001). La evaluación: ¿Un acto gozoso o doloroso? Revista de Educación, 290, 13-14.

Arce, C., Stem, D. E., Andrade, E. M. \& Seoane, G. (1997). Comparación de escalas de respuestas para la medición de las actitudes. Psicothema, 9, 541-545.

Asún, R. \& Zúñiga, C. (2008). Ventaja de los modelos politómicos de teoría de respuesta al ítem en la medición de actitudes sociales. El análisis de un caso. Psykhe, 17(2), 103-115.

Briñol, P., Horcajo, J., Becerra, A., Falces, C. \& Sierra, B. (2002). Cambio de actitudes implícitas. Psicothema, 14, 771-775.

Cárdenas, M. \& Barrientos, J. (2008). Actitudes explícitas e implícitas hacia los hombres homosexuales en una muestra de estudiantes universitarios en Chile. Psykhe, 17(2), 17-25.

Centro de Perfeccionamiento, Experimentación e Investigaciones Pedagógicas (2005). Instructivo: sistema de evaluación del desempeño docente. Santiago, Chile: Ministerio de Educación, Área de Acreditación y Evaluación Docente.

Centro de Perfeccionamiento, Experimentación e Investigaciones Pedagógicas (2008). Resultados evaluación docente 2008. Extraído el 11 Junio, 2008, de http://www.rmm.cl/index_sub.php?id_ contenido $=14197 \& i d \_p o r t a l=200 \& i d \_s e c c i o n=1186$

Cepeda, L., Espinoza, K. \& Rojas, A. (2003). Autoestima del profesor y desempeño docente, en profesores pertenecientes a establecimientos educacionales municipalizados de la comuna de Coquimbo. Tesis no publicada para optar al título de Psicólogo y al 
grado de Licenciado en Psicología, Universidad de La Serena, La Serena, Chile.

Cerda, J., Cortés, A., Mejías, B. \& Milla, M. (2006). Estudio descriptivo correlacional acerca de la autoestima del profesor y la actitud hacia la evaluación del desempeño docente en una muestra de cien profesores de las comunas de La Serena $y$ Coquimbo. Tesis no publicada para optar al título de Psicólogo y al grado de Licenciado en Psicología, Universidad de La Serena, La Serena, Chile.

Colegio de Profesores de Chile A.G. (2000). La complejidad de las políticas de evaluación del desempeño docente. Docencia, 10, 51-53.

Colegio de Profesores de Chile A.G. (2003). Fin de las calificaciones: Nuevo Sistema Nacional sobre Evaluación del Desempeño Docente. Docencia, 20, 80-87.

Cortés, F. (2007, Octubre). Planes de superación profesional: una alternativa de desarrollo profesional ligada a la evaluación docente. Ponencia presentada en la Mesa Redonda Impacto y consecuencias de la evaluación de profesores: presente y futuro. Seminario Evaluación Docente en Chile. Fundamentos, Experiencias y Resultados, MIDE UC, Centro de Medición de la Escuela de Psicología de la Pontificia Universidad Católica de Chile, Santiago, Chile. Extraído el 19 Agosto, 2008, de http://www.mideuc.cl/seminario/presentaciones. html

Chávez, M., Tron, R. \& Vaquero, E. (1992). Las escalas de actitudes y la sociometría en la práctica psicológica. En A. Silva (Ed.), Métodos cuantitativos en psicología: un enfoque metodológico (pp. 777-779). Ciudad de México: Trillas.

Chile, Ministerio de Educación (2000). Estándares de desempeño para la formación inicial de profesores. Santiago, Chile: Autor.

Chile, Ministerio de Educación (2003). Marco para la buena enseñanza. Santiago, Chile: Autor.

Chile, Ministerio de Educación (2004a, Febrero 12). Ley 19.993 - Otorga un mejoramiento especial a los profesionales de la educación que indica. Santiago, Chile: Autor. Extraído el 12 Mayo, 2008, de http:// www.mineduc.cl/biblio//documento/19933.pdf

Chile, Ministerio de Educación (2004b, Agosto 9). Ley 19.961 sobre evaluación docente. Santiago, Chile: Autor. Extraído el 10 Agosto, 2008, de http://www. docentemas.cl/documentos.php

Chile, Ministerio de Educación (2004c, Agosto 30). Aprueba Reglamento sobre Evaluación Docente. Decreto $N^{o}$ 192. Santiago, Chile: Autor, Departamento Jurídico. Extraído el 27 Junio, 2008, de http://www.docentemas.cl/documentos. php

Chile, Ministerio de Educación (2009, Marzo 24). Resultados evaluación docente 2008. Santiago, Chile: Autor. Extraído el 24 Agosto, 2009, de http:// www.docentemas.cl/docs/ResultadoEDocente2008. pdf

Danielson, Ch. (2007, Octubre). Una introducción a la evaluación de profesores. Presentación realizada en el Seminario Evaluación Docente en Chile.
Fundamentos, Experiencias y Resultados, MIDE UC, Centro de Medición de la Escuela de Psicología de la Pontificia Universidad Católica de Chile, Santiago, Chile. Extraído el 19 Agosto, 2008, de http://www.mideuc.cl/seminario/presentaciones. html

De Vicente, P. (1992). La adquisición del conocimiento pedagógico del contenido por una profesora principiante. En A. Estebaranz \& V. Sánchez (Eds.), Pensamiento de los profesores y desarrollo profesional. Tomo I Conocimiento y teorías implicitas (pp. 183-198). Sevilla: Servicio de Publicaciones de la Universidad de Sevilla.

De Vicente, P. (1996). Formación y evaluación basada en el centro. En A. Villa (Coord.), Evaluación de experiencias y tendencias en la formación del profesorado (pp. 289-332). Bilbao: Universidad de Deusto, Instituto de Ciencias de la Educación.

De Vicente, P. (2002). Desarrollo profesional del docente en un modelo colaborativo de evaluación. Bilbao: Universidad de Deusto, Instituto de Ciencias de la Educación.

Elliot, J. (1996). El cambio educativo desde la investigación acción. Madrid: Morata.

Hargreaves, A. (1996). Profesorado, cultura y postmodernidad. Madrid: Morata.

Manzi, J. (2007, Octubre). Evaluación docente: antecedentes, resultadosy proyecciones. Presentación realizada en el Seminario Evaluación Docente en Chile. Fundamentos, Experiencias y Resultados, MIDE UC, Centro de Medición de la Escuela de Psicología de la Pontificia Universidad Católica de Chile, Santiago, Chile. Extraído el 19 Agosto, 2008, de http://www.mideuc.cl/seminario/presentaciones. html

Marcelo, C. (1999). Formación del profesorado para el cambio educativo. Barcelona: Ediciones Universitarias de Barcelona.

Morera, D. \& Rodríguez, A. (1994). La relación entre actitudes y teorías implícitas: su estudio en situaciones de conflicto intergrupos. Psicothema, 6, 215-227.

Murillo, P. (1999). El aprendizaje del profesorado y los procesos de cambio. Sevilla: Mergabulum Edición y Comunicación.

Nevo, D. (1997). Evaluación basada en el centro: un diálogo para la mejora educativa. Bilbao: Ediciones Mensajero.

Nieto, J. (1996). La autoevaluación del profesor: como puede el profesor evaluar y mejorar su práctica docente. Barcelona: Wolters Kluwer.

Núñez, I. (2000). Lo que nos dice la historia: la evaluación de profesores en Chile. Docencia, 10, 70-79.

Reyes, E. (2000). Nivel de impacto profesional que tiene la evaluación del desempeño docente realizado por alumnos de séptimo a segundo medio en el Instituto Alonso de Ercilla de los Hermanos Maristas en las áreas de clima de trabajo, métodos de trabajo y actitud del profesor hacia el grupo. Tesis no publicada para optar al grado de Magíster en Educación, Mención Administración Educacional, Pontificia Universidad Católica de Chile, Santiago, Chile. 
Sierra Bravo, R. (1999). Técnicas de investigación social, teoría y ejercicios $\left(13^{\mathrm{a}}\right.$ ed.). Madrid: Parainfo.

Villar, L. M. (1991). Paradigmas de formación del profesorado. En A. Medina \& M. L. Sevillano (Eds.), Didáctica (pp. 499-526). Madrid: Universidad
Nacional de Educación a Distancia.

Villar, L. M. (Coord.) (1995). Un ciclo de enseñanza reflexiva. Bilbao: Ediciones Mensajero.

Yániz, C. (1998). Un sistema de evaluación y mejora para el profesorado de educación primaria. Bilbao: Ediciones Mensajero.

Fecha de recepción: Septiembre de 2008.

Fecha de aceptación: Septiembre de 2009. 